\title{
Metabolomics: Which Role in Asphyxia and Sepsis?
}

\section{Gabriele Finco', Paola Scano ${ }^{2}$, Ernesto d'Aloja ${ }^{3}$, Emanuela Locci ${ }^{3}$, Mario Musu', Paolo Mura', Vassilios Fanos ${ }^{4}$ and Antonio Noto ${ }^{4 *}$}

${ }^{1}$ Department of Anesthesiology, Cagliari University, Italy

${ }^{2}$ Department of Chemical and Geological Sciences, Cagliari University, Italy

${ }^{3}$ Department of Public Health, Clinical and Molecular Medicine-Forensic Science Unit-Cagliari University, Italy

${ }^{4}$ Department of Pediatrics and Clinical Medicine, Section of Neonatal Intensive Care Unit, Puericulture Institute and Neonatal Section, Cagliari University, Italy

\begin{abstract}
Metabolomics is a new "omics" approach concerning the high-throughput identification, quantification and characterization of endogenous and exogenous metabolites. This new technique is gaining consideration upon medical practice improving the single metabolite analysis as well as measuring the response to treatment in intensive care units. However, despite great efforts, very little research has new biomarkers or specific metabolic profiles, which characterize disorders such as asphyxia and sepsis. Here, we point out some of the recent works published in literature regarding the management of these conditions having high mortality and morbidity rates. The purpose of this review is also to highlight the ability of metabolomics to find early biomarkers for these conditions as well as to predict the development of sideleffects due to the therapy. The goal is to demonstrate that the metabolomics technique can be considered a viable option for the study of these conditions, which may help to develop a better care in intensive departments.
\end{abstract}

Keywords: Metabolomics; 1H-NMR; GC/MS; Anaesthesia; Resuscitation; Asphyxia; Sepsis

\section{Introduction}

Over the past years there has been an increased interest in encouraging research in the anaesthesia and resuscitation fields especially regarding topics that arise great interest for the national health system $[1,2]$. However, despite great efforts, very little research has been translated into the clinical practice. Among the recent technologies, metabolomics seems to be able to provide a remarkable impact trying to improve the diagnosing, prognosis and stratification risk of human diseases [3]. In this regard, the most studied pathologies concerning the intensive care units (ICU) are asphyxia and sepsis. The former, by means of perinatal asphyxia, is one of the main causes of neonatal death especially in developing countries. The incidence has been reported to be $2-6 / 1,000$ term births [4]. It is a condition in which there is a decrease of oxygen to the tissues, in spite of adequate blood flow, leading to a wide scale of injury, depending on several factors such as duration of insult, the recovery time after injury, and the intervention provided. Currently, a limited range of biochemical tests are in clinical use. Early biomarkers indicating the duration and severity of hypoxia and enabling risk stratification immediately after asphyxia might be particular helpful.

Another serious disorder, that is currently studied, is sepsis; it is one of the most common causes of death in ICU [5]. Sepsis condition affects annually over 750,000 people with high morbidity and mortality rates [6]. Recent studies estimate that the number of cases will reach almost one million by 2020 [7]. Sepsis in clinical terms is a systemic inflammatory response to a virus, bacteria or fungi infection, leading to a massive production of pro-inflammatory mediators. It leads to an alteration of metabolic balance, which results in tissue necrosis, hypotension, organ injury, and death [8]. In addition, among the sepsis-like syndrome there is a different rate of mortality ranging from $7 \%$ for the systemic inflammatory response syndrome (SIRS), $20 \%$ for the severe sepsis and $46 \%$ for the septic shock [9]. A special part is given to the neonatal sepsis, which is defined as a complex clinical syndrome and it is one of the most significant causes of preterm infant's morbidity and mortality. Recent studies have shown that more than $21 \%$ of very low birth weight (VLBW) infants surviving beyond 72 hours have at least one episode of blood culture-confirmed sepsis. Its incidence has a range between $0.5 \%$ and $1 \%$ in the developing countries and the timing-identification is a major diagnostic problem [10,11]. Currently, for most of biochemical and haematological tests, the range of false negative and false positive is still high [10].

This scenario shows, without any doubt, the need for new improvements for both asphyxia and sepsis in the early diagnosis as well as monitoring the risk stratification, complications and death. Based on this fact, metabolomics, analysing a whole metabolic profile, can help to improve the knowledge by defining patient's metabolic phenotype and discovering typical signatures representing the disease under investigation.

\section{The Metabolomics Technology}

The metabolomics approach consists on the quantitative analysis of large number of metabolites within a biological sample such as urine, blood, saliva etc. From a clinical point of view, the study of the metabolites within a biofluid is similar to observe an instantaneous metabolic snapshot, in fact, the metabolites $(<1000 \mathrm{Da})$ represent the end products of gene, transcripts and proteins, offering unique insights into small molecule regulation, which may uncover new biochemical patterns [12]. Metabolomics studies have taken advantage of advanced instrumentation such as nuclear magnetic resonance spectrometry (NMR), and mass spectrometry (MS) coupled with a multivariate statistical data analysis (MVA) [13]. NMR spectrometry is based on the magnetic properties of certain nuclei such as hydrogen, carbon

*Corresponding author: Antonio Noto, Department of Pediatrics and Clinica Medicine, Section of Neonatal Intensive Care Unit, Puericulture Institute and Neonatal Section, Cagliari University, Italy, Tel: 0039/3331014029; E-mail: antonionoto@hotmail.com

Received April 25, 2014; Accepted July 21, 2014; Published July 28, 2014

Citation: Finco G, Scano P, d'Aloja E, Locci E, Musu M, et al. (2014) Metabolomics: Which Role in Asphyxia and Sepsis?. J Anesth Clin Res 5: 420. doi:10.4172/21556148.1000420

Copyright: ( 2014 Finco G, et al. This is an open-access article distributed under the terms of the Creative Commons Attribution License, which permits unrestricted use, distribution, and reproduction in any medium, provided the original author and source are credited. 
and phosphorus. The technique is rapid and non-destructive, for the sample, it usually requires minimal laboratory preparation and it allows capturing simultaneously a large number of metabolites with an analytical sensitivity ranging between $\mathrm{mM} / \mathrm{L}$ and $\mu \mathrm{mol} / \mathrm{L}$; below this cut-off, the detection and quantification of metabolites are unreliable. However, for other molecules or toxic metabolites, the detection limit of the NMR may be inadequate. Therefore, a more sensitive technique needs to be used. MS is still considered the gold standard in metabolite detection. Its sensitivity allows detection of several hundreds of small molecules reaching concentration of $\mathrm{nmol} / \mathrm{L}$ range. Commonly, MS methods involve the separation of molecules by GC;or LC to unravel the metabolite complexity [14]. However, it requires longer analytical time, a derivatization process and the limitation of volatile compounds. Due to the huge diversity of chemical structures and the large differences in abundance, there is no single method available to analyse the entire metabolome. Therefore, a number of complementary approaches need to be used for detect as many metabolites as possible [15].

\section{Data Analysis}

The data obtained following the analysis are represented as a set of peaks. The intensities of the latter are proportional to the concentration of the metabolite. The introduction of a "standard" compound in known concentration is used as a reference to calculate the concentration of various metabolites in the biological fluid from the ratios of intensities. Subsequently, the analysis of the spectra requires the use of mathematical tools that are useful for extracthidden information among several thousands of variables [16,17]. The representation of data in spaces of small dimensions greatly facilitates the analysis and interpretation of results. MVA is classified into two categories: "unsupervised" and "supervised" methods [18,19]. A typical unsupervised approach is represented by the PCA (principal component analysis). It can be used to identify specific structures in a dataset such as clusters, anomalies or trends that exist between the observations. The PCA allows representing similarity between observations and variables. The analysed data are projected along the directions that allow obtaining the maximum possible variance. The first principal component is defined by the set of variables that describe most of the variance; the second describes the main component orthogonal to the first.

However, most of the predictive models rely on supervised models. Supervised methods require a training data set, in which the outcome is known, to build a predictive model. Samples belonging to the same group are close to each other and the resulting variables of importance (VIP) of the model can be considered as the weight factor that allows identifying the main metabolites responsible for the separation among the groups in the score plot. In general, it is essential to confirm the findings of any of these multivariate methods using a second set of "blind" samples, which may adequately test the results obtained. Once the best model has been built, the parameters $\mathrm{R}^{2}$ and $\mathrm{Q}^{2}$ should be tested. $\mathrm{R}^{2}$ estimates goodness of fit, $\mathrm{Q}^{2}$ estimates goodness of prediction. A high $\mathrm{R}^{2}$ close to 1 (or between 0.7 and 0.9 ) and high $\mathrm{Q}^{2}$ close to 0.8 (between 0.6 and 0.9 ) are evidences of an excellent mathematical model. This approach allows the classification of the different samples on the basis of metabolic profiles and to search for new biomarkers that characterize each group of samples under study. In this regard, it is always mandatory to reduce the number of false positive biomarkers assessing the accuracy of the multivariate model. Moreover, it must be aware that metabolomics studies are at risk for potential clinical confounders such as interindividual variability, diet, drug effects, age, sex, and comorbities. Therefore, each of these confounders needs to be taken into account.

\section{The Translation Role for Metabolomics Studies}

Once the identification of the new biomarkers is completed, it can be considered only the first step for the translation process. In fact, the typical flow "from laboratory to the patient bedside" involves several other steps that are called biomarker validation. It consists in the estimation of the performance in terms of sensitivity and specificity using a receiver operative characteristic (ROC) curve. The sensitivity is the percentage of subjects/patients who are correctly categorized with a biomarker among those who truly have the disease. Analogously, specificity is the percentage of subjects/patients who are categorized as not having the disease among all patients who truly don't have the disease. The area under the ROC (AUROC) provides a good measure of the overall model performance. An AUROC of 1 represents the ideal performance with a sensitivity and specificity of $100 \%$. Subsequently, the limit of detection, the robustness and the intra laboratory reproducibility are also parameters that need to be careful tested. Furthermore, the results achieved must be tested in different laboratories aiming to reproduce identical results independently.

\section{Metabolomics and Asphyxia}

Some papers dealing with the impact of asphyxia on global metabolism have recently appeared in the literature (Table 1). In particular, a work published by Vento et al. [20] explored the effect of resuscitation using pure oxygen (100\%) compared to the room air concentration (21\%) in a court of asphyctic term neonates compared to a control group [20]. In order to asses the presence of damage, authors measured oxidative stress biomarkers such as: reduced glutathione (GSH), oxidized glutathione and the superoxide dismutase (SOD), while to test the cardiac and renal damage they used plasma cardiac troponin $\mathrm{T}(\mathrm{c} \operatorname{TnT})$ and urinary $\mathrm{N}$-acetyl-glucosaminidase (NAG). Both groups of asphyctic neonates (100\%/21\%) showed an increased production of oxidative stress biomarkers, moreover, the group resuscitated with pure oxygen also revealed significantly higher value of cTnT and NAG. Authors suggest that the room air resuscitation causes less oxidative stress and damage to heart and kidney than pure oxygen.

In a recent work, Solberg et al. tried to determine the best oxygen concentration used as resuscitation procedure [21]. Authors used electrospray ionization with a tandem mass spectrometry (ESI MS/MS) and a liquid chromatography-tandem mass spectrometry (LC-MS/MS) to study the effect of hypoxia and reoxygenation in 33 piglets, which were divided in three groups and resuscitated with different oxygen concentrations. The first group had $21 \%$ of oxygen, the second group $100 \%$ for 15 minutes and then $21 \%$, the last one just $100 \%$. Authors hypothesized that the metabolites belonging to each group may uncover a maker of hypoxia as well enabling to find the best resuscitation procedure. These metabolites included several key components of Krebs cycle metabolism, glycine and amino acid metabolisms. They suggested that the resuscitation with $100 \%$ of oxygen was correlated to the reduction, in terms of concentration, of the metabolites alphaketo-glutarate, succinate and fumarate, which was also associated to the delay in recovery.

In the following paper, Fanos et al. performed a comparative study on the metabolic effect on 40 piglets subjected to experimental hypoxia and reoxygenation [1]. Aim of this work was to investigate whether different metabolomics profiles occurred according to different oxygen concentrations administered at resuscitation $(18 \%, 21 \%, 40 \%$ and $100 \%)$. Urine metabolic profiles were analyzed at baseline and after reoxygenation by ${ }^{1} \mathrm{H}-\mathrm{NMR}$. The metabolites were identified by MVA 
Citation: Finco G, Scano P, d'Aloja E, Locci E, Musu M, et al. (2014) Metabolomics: Which Role in Asphyxia and Sepsis?. J Anesth Clin Res 5: 420. doi:10.4172/2155-6148.1000420

Page 3 of 5

\begin{tabular}{|l|l|l|l|l|}
\hline \multicolumn{2}{|l|}{ Summary of the metabolomics analysis for the asphyxia condition } \\
\hline $\begin{array}{l}\text { Approach (Un targeted/ } \\
\text { Targeted) }\end{array}$ & Analytical techniques & $\begin{array}{l}\text { Animal/Human } \\
\text { sampling }\end{array}$ & Biofluid & Key metabolic pathways \\
\hline Targeted & $\begin{array}{l}\text { Spectrophotometry } \\
\text { HPLC }\end{array}$ & Human (Newborns) & Blood & $\begin{array}{l}\text { Production of oxidative stress biomarkers, and significantly Vento et al. [20] } \\
\text { higher value of cTnT and NAG. }\end{array}$ \\
\hline Targeted & $\begin{array}{l}\text { ESI MS/MS } \\
\text { LC-MS/MS }\end{array}$ & $\begin{array}{l}\text { Animal } \\
\text { (Piglets) }\end{array}$ & $\begin{array}{l}\text { Plasma } \\
\text { Animal } \\
\text { (Piglets) }\end{array}$ & $\begin{array}{l}\text { The metabolites key components of Krebs cycle metabolism, Solberg et al. [21] } \\
\text { glycine and amino acid metabolisms. }\end{array}$ \\
\hline Untargeted & NMR/MVA & $\begin{array}{l}\text { Resuscitation with lower oxygen concentration was associated Fanos et al. [22] } \\
\text { with cellular homeostasis, maintenance, and carbohydrates } \\
\text { metabolisms. } \\
\text { Higher oxygen concentration was characterized by ROS } \\
\text { production. }\end{array}$ \\
\hline
\end{tabular}

Table 1: A schematic representation of metabolomics workflow applied to asphyxia samples.

\begin{tabular}{|c|c|c|c|c|c|c|}
\hline \multicolumn{7}{|c|}{ Summary of the metabolomics analysis for the sepsis condition } \\
\hline $\begin{array}{c}\text { Approach (Un } \\
\text { targeted/Targeted) }\end{array}$ & $\begin{array}{l}\text { Analytical } \\
\text { techniques }\end{array}$ & $\begin{array}{l}\text { Animal/Human } \\
\text { sampling }\end{array}$ & Biofluid & MVA & Key metabolites identified & Reference \\
\hline Untargeted & NMR & $\begin{array}{l}\text { Animal (Sprague- } \\
\text { Dawley rats) }\end{array}$ & Serum & $\begin{array}{l}\text { Orthogonal partial } \\
\text { least squares }\end{array}$ & $\begin{array}{l}\text { lactate, alanine, acetate, acetoacetate, } \\
\text { hydroxybutyrate, and formate }\end{array}$ & Lin et al. [23] \\
\hline Targeted & UPLC-Q-TOF-MS & $\begin{array}{l}\text { Animal (Sprague- } \\
\text { Dawley rats) }\end{array}$ & Plasma & $\begin{array}{l}\text { Orthogonal partial } \\
\text { least squares }\end{array}$ & $\begin{array}{l}\text { hypoxanthine, indoxylsufate, glucuronic } \\
\text { acid, gluconic acid, proline, uracil, } \\
\text { nitrotyrosine, uric acid, and trihydroxy } \\
\text { cholanoic acid }\end{array}$ & Liu et al. [24] \\
\hline Untargeted & NMR & $\begin{array}{l}\text { Animal (Sprague- } \\
\text { Dawley rats) }\end{array}$ & $\begin{array}{l}\text { Broncho- } \\
\text { alveolar lavage } \\
\text { fluid (BALF), } \\
\text { lung tissue and } \\
\text { serum }\end{array}$ & $\begin{array}{l}\text { Partial least squares- } \\
\text { discriminant analysis }\end{array}$ & $\begin{array}{l}\text { alanine, creatine, phosphoethanolamine, } \\
\text { and myoinositol increased in lung tissue; } \\
\text { creatine increased and myoinositol } \\
\text { decreased in balf; alanine, creatine, } \\
\text { phosphoethanolamine, and acetoacetate } \\
\text { increased in serum. }\end{array}$ & $\begin{array}{l}\text { Izquierdo-Garcia } \\
\text { et al. [25] }\end{array}$ \\
\hline $\begin{array}{l}\text { Untargeted } \\
\text { Targeted }\end{array}$ & $\begin{array}{l}\text { NMR } \\
\text { GC/MS }\end{array}$ & Human (Newborns) & Urine & $\begin{array}{l}\text { Orthogonal partial } \\
\text { least squares- } \\
\text { discriminant analysis }\end{array}$ & $\begin{array}{l}\text { NMR: acetate } \\
\text { acetone, citrate } \\
\text { creatinine glycine } \\
\text { lactate, lysine } \\
\text { glucose } \\
\text { GC/MS: lactate, glucose,, maltose, } \\
\text { ribitol, ribonic acid, pseudo-uridine, } \\
\text { 2,3,4-trihydroxybutyric acid, } \\
\text { 2-ketogluconic acid } \\
\text { 3,4-dihydroxybutanoic acid, } \\
\text { 3,4,5-trihydroxypentanoic acid }\end{array}$ & Fanos et al. [22] \\
\hline Untargeted & NMR & $\begin{array}{l}\text { Human } \\
\text { (Adults) }\end{array}$ & Serum & $\begin{array}{l}\text { Orthogonal partial } \\
\text { least squares }\end{array}$ & $\begin{array}{l}\text { glucose, 3-hydroxybutyrate, } \\
\text { o-acetylcarnitine, succinate, creatine, } \\
\text { and creatine phosphate lactate, } \\
\text { 2-hydroxyisovalerate, isobutyrate, } \\
\text { creatinine, trimethylamine n-oxide, and } \\
\text { urea branched-chain amino acids }\end{array}$ & Mickiewicz et al. [28] \\
\hline
\end{tabular}

Table 2: A schematic representation of metabolomics workflow applied to sepsis biofluids.

indicating that resuscitation with $21 \%$ of oxygen seems to be optimal in terms of survival, and rapidity of resuscitation. Authors also suggested that metabolomics approach could be a new tool for a more in-depth knowledge of the pathophysiological responses of the new-borns to the resuscitation with different oxygen concentration.

This recent literature suggests that metabolomics is a promising method to assess the best oxygen concentration to be used for resuscitation. Indeed, this approach is a powerful instrument of knowledge about the factors responsible of the metabolic modifications, which can help identifying metabolic patterns of function, disease, and injury, as well as for therapeutic strategies.

\section{Metabolomics and Sepsis}

Since sepsis is a complex multiorgan dysfunction, resulting in large changes in the organism metabolites, the analysis of the metabolome may be an attractive methodology for the determination of multiple metabolites. However, very little research has been performed using the metabolomics approach in the field of sepsis especially in human [22]. So far, only few recent papers have been published using both animal models, and human samples. These works are highlighted below (Table 2).

In 2009, Lin et al. [23] proposed an NMR-based metabolomics study to evaluate the feasibility to build and early predictive experimental model for the investigation of the sepsis syndrome. Authors advised that the purpose for performing this study was the lack of sensitivity of the common biomarkers, such as $\mathrm{C}$ reactive protein and procalcitonin, which are routinely used in clinical laboratory. By contrast, they hypothesized that a panel of metabolites could better identify an early septic condition, becoming in the next future a valid alternative. In order to perform this study, serum samples were collected from 60 male pathogen-free Sprague-Dawley rats (220-250 g; 6-8 weeks). The experimental sepsis was induced by cecal ligation and puncture (CLP) and rats were randomized into a sham-operated group (n 20) and a CLP group ( $\mathrm{n} 40$ ). According to the difference of survival during the following days, CLP rats were then divided into a surviving group (n 19 , in case of survival duration exceeding 6 days) and a nonsurviving group (n 21, survival duration between $24 \mathrm{~h}$ and 6 days). From each animal, approximately $1.0 \mathrm{~mL}$ of blood was collected by tail (sera were 
obtained by centrifugation at $3000 \mathrm{rpm}$ for $15 \mathrm{~min}$ ). Later, samples were stored at minus $80^{\circ} \mathrm{C}$ until NMR analysis. The metabolomics analysis revealed that the levels of six metabolites, mainly involved in energy metabolism, including lactate, alanine, acetate, acetoacetate, hydroxybutyrate, and formate, changed markedly in septic rats and especially in the nonsurvivors group. Using these metabolites, a predictive model called radial basis function neural network was created for an early prognostic evaluation of sepsis. The model achieved a prediction accuracy of approximately $87 \%$ indicating that the NMR-based metabonomic approach may be a promising technique for an early prognosis with the advantages of being rapid, less costly, and more efficient. Authors stated that the metabolomics approach would offer in the near future a promising tool for clinicians to predict the outcome of septic patients in intensive care unit.

More recently, Liu et al. [24] investigated the sepsis condition caused by a thermally injured in experimental animal model of rats. Authors, using ultra performance liquid chromatographyquadrupole time of flight mass spectrometry (UPLC-Q-TOF-MS) on plasma samples, were able to identify 9 metabolites, which were responsible for the septicemia. The metabolites related to the sepsis were: hypoxanthine, indoxylsufate, glucuronic acid, gluconic acid, proline, uracil, nitrotyrosine, uric acid, and trihydroxy cholanoic acid. A deeper analysis of these metabolites suggested their involvement in oxidative stress and tissue damage. In another work, Izquierdo-Garcia et al. [25] has used NMR spectroscopy coupled with MVA for the study of sepsis. They collected and analyzed bronchoalveolar lavage fluid (BALF), lung tissue and serum samples from 28 male Sprague Dawley rats, which were divided into two groups. The first group had the cecal ligation puncture in order to develop sepsis infection, while the second group did not receive the puncture. Using the metabolomic approach the authors were able to construct a predictive model, which could discriminate between the two groups with a sensitivity and specificity of $100 \%$. The most important metabolites in the septic rat's model were: alanine, creatine, phosphoethanolamine, and myoinositol increased in lung tissue; creatine increased and myoinositol decreased in BAL fluid; and alanine, creatine, phosphoethanolamine, and acetoacetate increased in serum.

The first report on early and late diagnosis of neonatal sepsis was performed by Fanos et al. [2]. In this study, authors used a combined approach based on both ${ }^{1} \mathrm{H}-\mathrm{NMR}$ and gas-chromatography-mass spectrometry (GC-MS) techniques, coupled with MVA, to investigate the impact of sepsis in a population of neonates. The aim of this study was to evaluate the capability of the metabolomics approach to identify a potential metabolic profile related to the neonatal septic condition. The population includes 25 neonates and among them, 9 patients had a diagnosis of sepsis while 16 were controls. ${ }^{1} \mathrm{H}-\mathrm{NMR}$ and GC/MS spectra were acquired for all urine samples and analysed by using an Orthogonal Partial Least Square- Discriminant Analysis (OPLS-DA). The OPLS-DA model showed a significant separation between the two groups of samples, and the metabolites responsible for the separation were identified. In particular, the infected group was characterized by a urinary increase in acetate, acetone, glycine, lysine lactate, glucose, maltose, and a decrease of ribitol, ribonic acid, pseudo uridine, 2,3,4-trihydroxybutyric acid 2-ketogluconic acid 3,4-dihydroxybutanoic acid 3,4,5-trihydroxypentanoic acid, citrate and creatinine. The authors indicate that was possible to identify by MVA a different metabolomics pattern between classes of neonates having a different clinical outcome. These results agree with recent works published in the literature. In particular, the presence of acetone in the urine of the septic group has been suggested as a compensatory reaction to a reduced level of ATP. Lactate and glucose were also found to be increased in the urine of septic patients compared to controls, while citrate, a metabolite of the Krebs cycle, had decreased [15,26,27]. This study showed a unique metabolic profile of the patients affected by sepsis compared to non-affected ones, with a statistically significant difference between the two groups ( $\mathrm{p}=0.05)$.

At the same time, Mickiewicz et al. [28] studied the metabolic profiles of human serum sample using ${ }^{1} \mathrm{H}$ NMR metabolomics approach as tool for septic shock diagnosis. Based on the idea that metabolomics can provide a unique insight into metabolic changes of living systems, the aim of the study was focused on developing a new approach to improve the diagnosis and prognosis of sepsis. Human blood samples from 39 septic shock patients and 20 controls were collected within the first 24 hours after admission to the ICU. The patients recruited were in accordance to specific published criteria for SIRS and septic shock. The samples were then centrifuged at $1,200 \times \mathrm{g}$ for 10 minutes, collected in a $15-\mathrm{mL}$ tube, and frozen at $-80^{\circ} \mathrm{C}$ until NMR analysis. In order to prepare samples for NMR experiment, the sera were thawed and filtered twice using $3-\mathrm{kDa}$ filters. The resulting spectra were first processed using an unsupervised method, which was able to separate the septic shock samples and controls without any outliers. Subsequently, to better reveal the differences between the metabolic profiles of the two groups, a supervised OPLS-DA analysis was performed. The most important metabolites responsible for the clustering between the groups were identified. Sixty metabolites changed significantly in septic shock patients, they are mainly involved in energy metabolism such as glucose, 3-hydroxybutyrate, O-acetylcarnitine, succinate, creatine, and creatine phosphate. Authors also suggested that the significant increases in the concentration of metabolites such as lactate, 2-hydroxyisovalerate, isobutyrate, creatinine, trimethylamine $\mathrm{N}$-oxide, and urea may be related to organ failure. In addition, the levels of branched-chain amino acids decrease significantly in the sepsis group, suggesting an increased protein breakdown and oxidation as source for the energy production. A very remarkable goal of the study is the demonstration of a strong potential prediction of mortality among the septic shock patients. The metabolomics approach appeared more efficient than the common scores (APACHE/SOFA) for the early prognosis of patient outcome. Nonetheless, authors considered the study as an initial step for the use of the metabolomics approach in a clinical setting. In fact they underlined the need for further studied across multiple centres.

\section{Conclusions}

So far, it can be stated that the metabolomics technique can be considered a viable option for the study of pathologies such as asphyxia and sepsis. Certainly, further studies are requested to confirm these preliminary results; nevertheless, very interesting data are available in the literature.

\section{References}

1. Fanos V, Noto A, Xanthos T, Lussu M, Murgia F, et al. (2014) Metabolomics network characterization of resuscitation after normocapnic hypoxia in a newborn piglet model supports the hypothesis that room air is better. Biomed Res Int 2014: 731620.

2. Fanos V, Caboni P, Corsello G, Stronati M, Gazzolo D, et al. (2014) Urinary (1) H-NMR and GC-MS metabolomics predicts early and late onset neonatal sepsis. Early Hum Dev 90 Suppl 1: S78-83.

3. Nicholson JK, Lindon JC, Holmes E (1999) 'Metabonomics': understanding the metabolic responses of living systems to pathophysiological stimul via multivariate statistical analysis of biological NMR spectroscopic data Xenobiotica 29: 1181-1189. 
Citation: Finco G, Scano P, d'Aloja E, Locci E, Musu M, et al. (2014) Metabolomics: Which Role in Asphyxia and Sepsis?. J Anesth Clin Res 5: 420. doi:10.4172/2155-6148.1000420

Page 5 of 5

4. Saugstad OD (2011) Reducing global neonatal mortality is possible. Neonatology 99: 250-257.

5. Nee PA (2006) Critical care in the emergency department: severe sepsis and septic shock. Emerg Med J 23: 713-717.

6. Angus DC, Linde-Zwirble WT, Lidicker J, Clermont G, Carcillo J, et al. (2001) Epidemiology of severe sepsis in the United States: analysis of incidence, outcome, and associated costs of care. Crit Care Med 29: 1303-1310.

7. Namas R, Zamora R, Namas R, An G, Doyle J, et al. (2012) Sepsis: Something old, something new, and a systems view. J Crit Care 27: 314.

8. Levy MM, Fink MP, Marshall JC, Abraham E, Angus D, et al. (2003) 2001 SCCM/ESICM/ACCP/ATS/SIS International Sepsis Definitions Conference. Crit Care Med 31: 1250-1256.

9. Rangel-Frausto MS, Pittet D, Costigan M, Hwang T, Davis CS, et al. (1995) The natural history of the systemic inflammatory response syndrome (SIRS). A prospective study. JAMA 273: 117-123.

10. Mussap M, Fanos V (2011) Neonatal nephrology and laboratory medicine: an effective interdisciplinary model to improve the outcome in neonatal intensive care unit. J Matern Fetal Neonatal Med 24 Suppl 2: 1-2.

11. Mussap M, Degrandi R, Cataldi L, Fanos V, Plebani M (2007) Biochemica markers for the early assessment of neonatal sepsis: the role of procalcitonin. J Chemother 19 Suppl 2: 35-38.

12. lacovidou N, Syggelou A, Chalkias A, Atzori L, Xanthos T, et al. (2014) Metabolomics applied in neonatology. Bioanalysis 6: 403-410.

13. Dunn WB, Broadhurst DI, Atherton HJ, Goodacre R, Griffin JL (2011) Systems level studies of mammalian metabolomes: the roles of mass spectrometry and nuclear magnetic resonance spectroscopy. Chem Soc Rev 40: 387-426.

14. Wishart DS, Knox C, Guo AC, Eisner R, Young N, et al. (2009) HMDB: a knowledgebase for the human metabolome. Nucleic Acids Res 37: D603-610.

15. Mussap M, Antonucci R, Noto A, Fanos V (2013) The role of metabolomics in neonatal and pediatric laboratory medicine. Clin Chim Acta 426: 127-138.

16. Weljie AM, Newton J, Mercier P, Carlson E, Slupsky CM (2006) Targeted profiling: quantitative analysis of $1 \mathrm{H}$ NMR metabolomics data. Anal Chem 78 . 4430-4442.

17. Issaq HJ, Van QN, Waybright TJ, Muschik GM, Veenstra TD (2009) Analytical and statistical approaches to metabolomics research. J Sep Sci 32: 2183-2199.

18. Brereton RG (2009) Chemometrics for patter recognition. New York: Wiley and Sons, Inc.

19. Dettmer K, Aronov PA, Hammock BD (2007) Mass spectrometry-based metabolomics. Mass Spectrom Rev 26: 51-78.

20. Vento M, Sastre J, Asensi MA, Viña J (2005) Room-air resuscitation causes less damage to heart and kidney than $100 \%$ oxygen. Am J Respir Crit Care Med 172: 1393-1398.

21. Solberg R, Enot D, Deigner HP, Koal T, Scholl-Bürgi S, et al. (2010) Metabolomic analyses of plasma reveals new insights into asphyxia and resuscitation in pigs. PLoS One 5: e9606.

22. Fanos V, Barberini L, Antonucci R, Atzori L (2012) Pharma-metabolomics in neonatology: is it a dream or a fact? Curr Pharm Des 18: 2996-3006.

23. Lin ZY, Xu PB, Yan SK, Meng HB, Yang GJ, et al. (2009) A metabonomic approach to early prognostic evaluation of experimental sepsis by (1)H NMR and pattern recognition. NMR Biomed 22: 601-608.

24. Liu XR, Zheng XF, Ji SZ, Lv YH, Zheng DY, et al. (2010) Metabolomic analysis of thermally injured and/or septic rats. Burns 36: 992-998.

25. Izquierdo-García JL, Nin N, Ruíz-Cabello J, Rojas Y, de Paula M, et al. (2011) A metabolomic approach for diagnosis of experimental sepsis. Intensive Care Med.

26. Wolfe RR, Shaw JH, Durkot MJ (1983) Energy metabolism in trauma and sepsis: the role of fat. Prog Clin Biol Res 111: 89-109.

27. Mickiewicz B, Vogel HJ, Wong HR, Winston BW (2013) Metabolomics as novel approach for early diagnosis of pediatric septic shock and its mortality. Am J Respir Crit Care Med 187: 967-976.

28. Mickiewicz B, Duggan GE, Winston BW, Doig C, Kubes P, et al. (2014) Metabolic profiling of serum samples by $1 \mathrm{H}$ nuclear magnetic resonance spectroscopy as a potential diagnostic approach for septic shock. Crit Care Med 42: 1140-1149.
Citation: Finco G, Scano P, d'Aloja E, Locci E, Musu M, et al. (2014) Metabolomics: Which Role in Asphyxia and Sepsis? J Anesth Clin Res 5: 420. doi:10.4172/2155-6148.1000420
Submit your next manuscript and get advantages of OMICS Group submissions

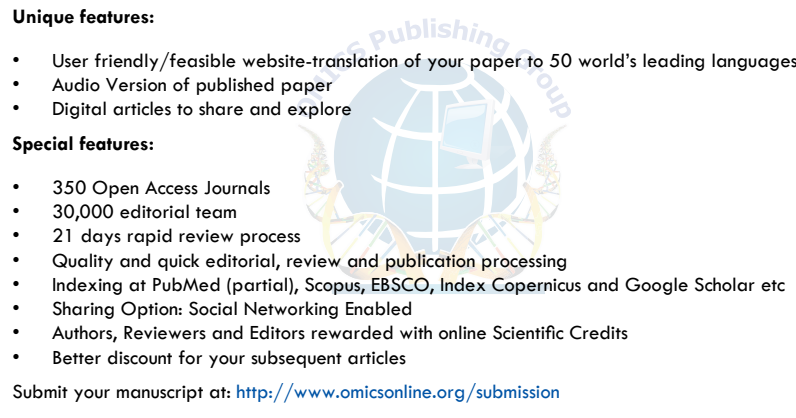

